\title{
1. Aeskulap-Forum: Ganzheitliche Therapien bei chronischen Schmerzen
}

\author{
Fortbildung für Ärztinnen und Ärzte, Aeskulap-Klinik Brunnen, 22. Oktober 2009
}

$\mathrm{D}$ er Begriff Schmerz beschreibt ein weites Spektrum von unangenehmen körperlichen Erfahrungen, Empfindungen und Gefühlen. In jedem Fall ist Schmerz als Signal beziehungsweise Symptom einer körperlichen oder psychischen Störung zu sehen.

Antike Hochkulturen und Naturvölker unterschieden Schmerzen nach der für das Auge sichtbaren Ursache. Unsichtbare Schmerzen und Schmerzen unbekannter Herkunft führte man auf böse Geister und übernatürliche Kräfte zurück. In der hippokratischen Medizin (400 v.Chr.) begann man Schmerzen als Ungleichgewicht der Körpersäfte zu sehen. Der römische Arzt Galen (129200 n.Chr.) differenzierte zwischen inneren und äusseren Schmerzen. Er nahm an, dass sich Körper und Seele gegenseitig beeinflussen. Das Verspüren von inneren Schmerzen führte er dabei auf eine fehlerhafte Zusammensetzung der Körpersäfte zurück. Die naturwissenschaftlich orientierte Medizin am Übergang zur Neuzeit reduzierte Schmerz auf ein rein körperliches Phänomen; Körper und Seele funktionieren in diesem Weltbild unabhängig voneinander. Im Lauf des 20. Jahrhunderts akzeptierte man, dass beim Verspüren von Schmerz auch biologische, psychologische und soziale Faktoren eine grosse Rolle spielen.

Schmerzen, die nicht rechtzeitig oder nur ungenügend behandelt werden, können sich zu einer Schmerzkrankheit entwickeln. Sensibilisierungen innerhalb des Nervensystems bewirken, dass leichte Reize, die normalerweise nicht als Schmerz gewertet werden, starke Schmerzempfindungen auslösen. Diese Sensibilisierungen können auf allen Stufen des peripheren, zentralen und autonomen Nervensys- tems entstehen und müssen bei einer patientengerechten Schmerztherapie in Betracht gezogen werden, bekräftigte Dr.med. Cesar Winnicki, Chefarzt der Aeskulap-Klinik in Brunnen, in seiner Begrüssung gegenüber den TeilnehmerInnen des Symposiums. Denn viele Faktoren können auf die Schmerzschwelle sowohl einen positiven als auch einen negativen Einfluss haben. Dazu gehören z.B. Schlaflosigkeit, Sorgen, Angst, Traurigkeit, Introversion, Depression, soziale Abhängigkeit, Langeweile und Isolation. Gefragt sind daher Therapiekonzepte mit einem ganzheitlichen Ansatz.

\section{Neuraltherapie}

Chronische Schmerzkrankheiten nehmen stark zu. Hoffnungen in nebenwirkungsarme medikamentöse Therapien haben sich zum Teil zerschlagen (Cox-2-Hemmer), und somit sind auch andere Therapie-Optionen gefragt. Eine aus Sicht der modernen Schmerzphysiologie logische Möglichkeit ist die Neuraltherapie. Aufgrund ihrer Leistungsfähigkeit lässt sie sich isoliert anwenden, passt jedoch auch in jedes interdisziplinäre Therapiekonzept.

Die Neuraltherapie nach Huneke ist eine Injektionsbehandlung, bei der Lokalanästhetika zur Diagnostik und Therapie genutzt werden. Therapeutisch werden gezielt neurophysiologische Reiz- und Hemm-Mechanismen angesprochen, welche bewirken, dass die Systeme autoregulatorisch den physiologischen Zustand anstreben. Die Behandlungsmethode, so die Ausführungen von Univ.Doz.Dr.med. Lorenz Fischer, Kollegiale Instanz für Komplementärmedizin an der Universität Bern, nutzt die regulatorischen Eigenschaften insbesondere des autonomen Nervensystems auf zwei unterschiedlichen Ebenen: zum einen über die Segmentreflektorik (peripher-spinale Ebene), zum andern über das sogenannte Störfeld. Ein Störfeld ist nach Huneke ein asymptomatischer, chronischer Reizzustand einer Struktur des Organismus. Häufig sind dies beispielsweise pathologische Veränderungen im ZahnKiefer-Bereich oder chronische Nebenhöhlenentzündungen usw. Als Folge dieses Reizzustandes kann jedes andere System des Organismus beeinträchtigt werden. Ein grosser Teil der diagnostischen und therapeutischen Arbeit mit Lokalanästhetika im lokalen und segmentalen Bereich (z.B. Injektionen an Nerven, vegetative Ganglien, Gelenke, myofasciale Triggerpunkte) wird auch in der konventionellen Medizin durchgeführt (Schmerzkliniken, Rheumatologen). In diesem punktuellen Bereich gibt es bezüglich Techniken und Indikationen keinen Unterschied zwischen konventioneller Medizin und Neuraltherapie.

In seinem Vortrag stellte Dr. Fischer die Schmerzmechanismen und darauf aufbauend mögliche neuraltherapeutische Interventionen dar. An verschiedenen Fallbeispielen wurden die verblüffenden Langzeiterfolge mit Neuraltherapie insbesondere bei austherapierten, chronischen Schmerzpatienten gezeigt, und die dargestellten Erfolge konnten in verschiedenen klinischen Studien bestätigt werden. Eine der wichtigsten Studien bei chronischen Schmerzen am Bewegungsapparat: J. Mermod, L. Fischer, L. Staub, A. Busato: Patient satisfaction of primary care for musculoskeletal diseases: A comparison between Neural Therapy 


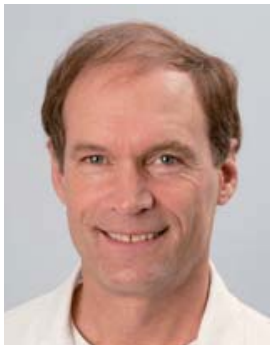

Dr.med. Daniel Friedli, Schwyz

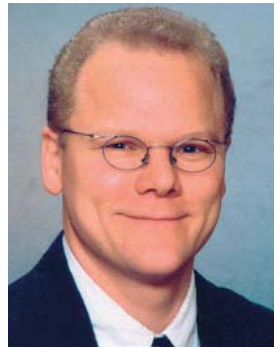

Dr.med. Hans P. Ogal, Brunnen

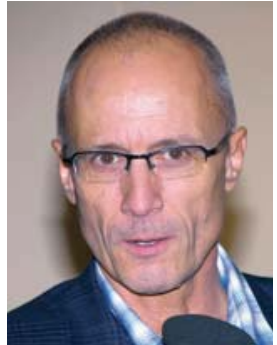

Dr.med. Lorenz Fischer, Bern

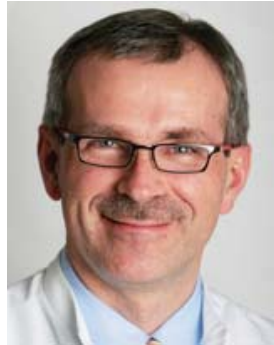

Dr.med. Cesar Winnicki, Brunnen

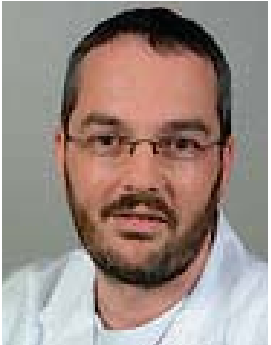

Prof.Dr.med. Christoph Konrad, Luzern

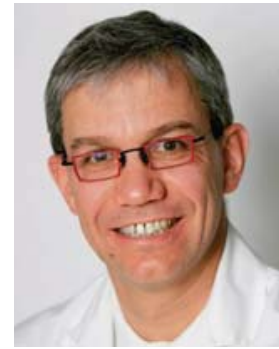

Prof.Dr.med.dent. Chris tian Besimo, Brunnen and conventional medicine. BMC 2008, 8: 33 (www.biomedcentral.com/1472$6882 / 8 / 33)$. Sie zeigt bessere Behandlungsresultate bei Patienten, welche mit Neuraltherapie behandelt wurden (gegenüber konventioneller Behandlung). In der Neuraltherapie-Gruppe mussten auch weniger Arbeitsunfähigkeiten verschrieben werden.

\section{Interventionelle Verfahren}

Die Therapie der Befindlichkeitsstörung Schmerz gehört prinzipiell zu den grundlegendsten Fertigkeiten eines jeden Arztes. Dies gelingt bei gut 70\% der Patienten zufriedenstellend. Bei den verbleibenden $30 \%$ ist die Unzufriedenheit der Patienten bei akuten und vor allem chronischen Schmerzen mit der angebotenen Schmerztherapie hoch, wie die Erfahrung von Dr.med. Daniel Friedli, Abteilung für Anästhesie am Spital Schwyz, zeigt.

Die interventionelle Schmerztherapie kommt bei etwa $2-3 \%$ der chronischen Schmerzpatienten zum Einsatz.
Sie wird im WHO-Stufenplan der malignen Schmerztherapie frühzeitig vorgeschlagen, ist einfach durchzuführen und nimmt in Anbetracht von zunehmender Medikamenten-Unverträglichkeit resp. Polipragmasie ständig an Bedeutung zu. Sie kann vereinfacht in 4 Gruppen eingeteilt werden:

1. Diagnostische Verfahren: Nervenund Gelenksinfiltrationen zur Lokalisierung des vermeintlichen Ursprungs der Schmerzen. Dank hochauflösender Ultraschalltechnologie und Röntgenbilddiagnostik können gezielte Nervenblockaden durchgeführt werden, welche in der weiteren Schmerztherapie extrem hilfreich sind.

\section{Schmerzmodulierende Verfahren:} Dazu gehören die repetitiven gezielten Infiltrationen (z.B. Stellatumblockade, selektive Nervenblockaden) und die sog. Field-Blocks, welche aufgrund der rep. Leitungsblockaden zum Teil die neuronale Funktion alterieren oder indirekt das Schmerzgedächtnis modifizieren. Die implantierbare periphere Nervenstimulation oder Rückenmarkstimulation (SCS) ist bei genau ausge- wählten Patienten äusserst wirkungsvoll, der genaue Mechanismus aber noch nicht vollständig bekannt. Mit der Deep Brain Stimulation wird diese Technik auch im Cerebrum angewendet. Die gepulste Radiofrequenz resp. die Cryoneurolyse kann zu den modulierenden Verfahren gezählt werden, zumal die Regenerationsfähigkeit der Nervenzellen vorhanden ist.

3. Destruierende Verfahren bei Patienten mit Schmerzen im terminalen Stadium: Lytische Substanzen wie Alkohol oder Phenol können in jedem Bereich der Nervenbahnen Einsatz finden. Cordotomien im Bereich des Tractus spinothalamicus finden selten eine Indikation bei unliateralen Schmerzen von terminalen Patienten. Radiofrequenztherapie mit Hitzeläsionen bis $80^{\circ} \mathrm{C}$ hat je nach Zentrum ein breites Indikationengebiet.

4. Implantable Drug Delivery System (IDDS): Die Indikation zur Implantation einer meist intrathekal applizierenden Medikamentenpumpe ist zur Behandlung von chronischen Schmerzzuständen vorgängig genauestens zu hinter-

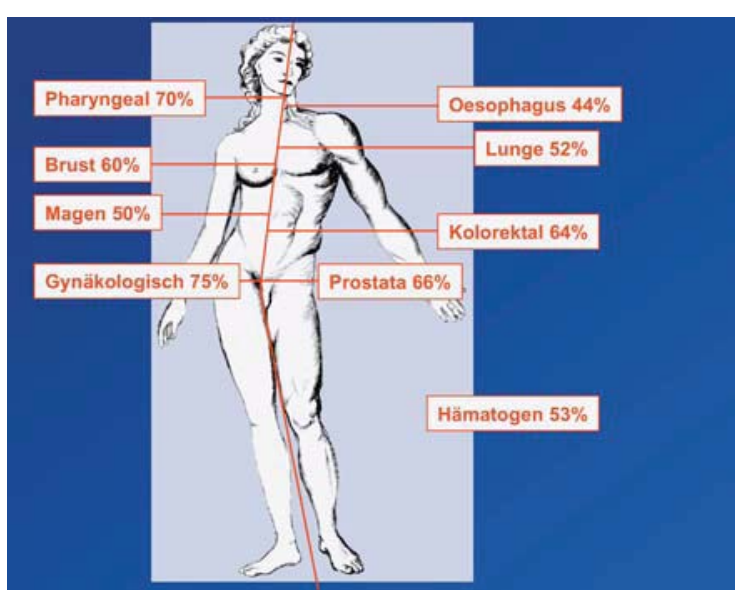

Anteil der Tumorpatienten mit Schmerzen (nach Tumorart)

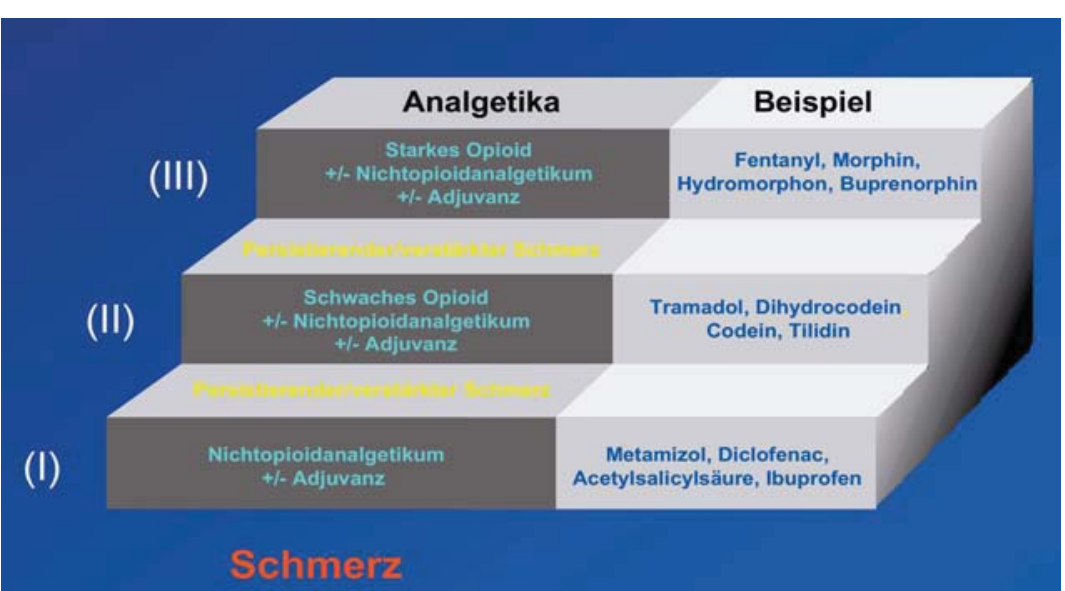

WHO Stufenschema der Schmerztherapie 


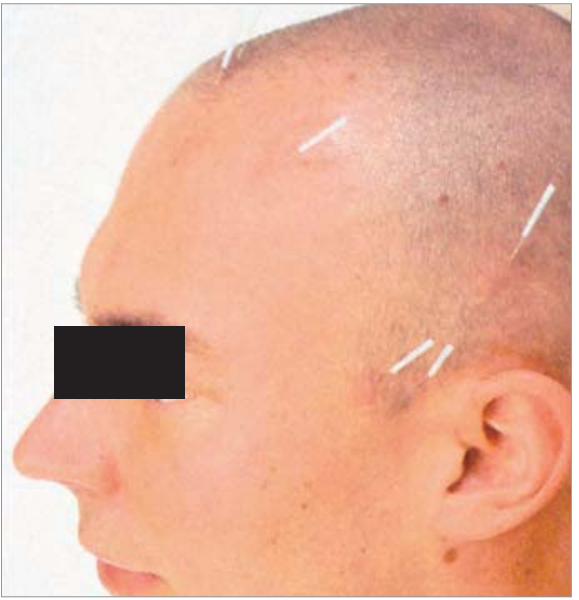

Schädelakupunktur nach Yamamoto (YNSA)

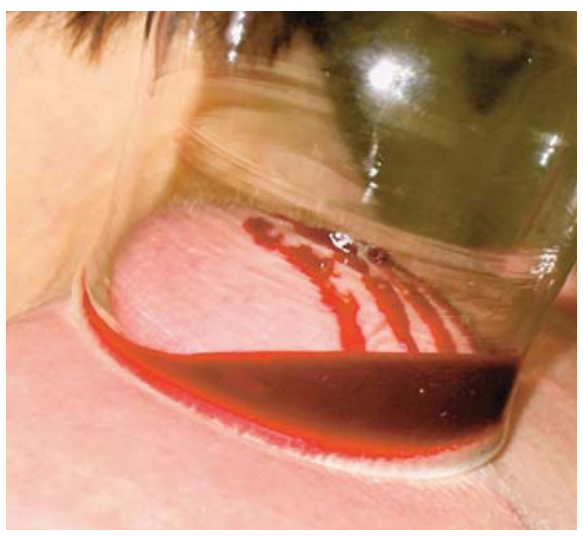

Blutiges Schröpfen im Bereich des M. trapezius

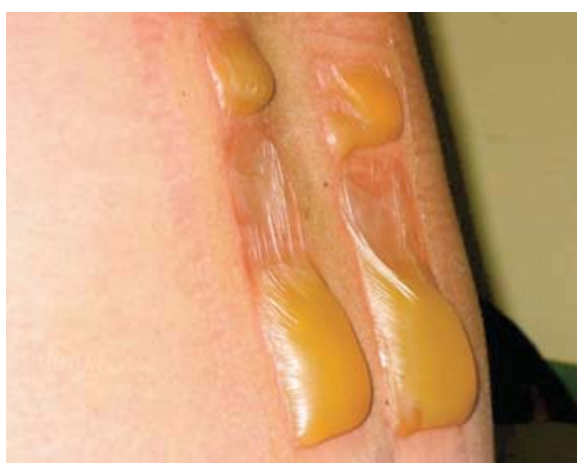

Cantharidenpflaster über dem lleosakralgelenk fragen. Bei der Therapie von zentraler Spastizität hat sich das System durchgesetzt.

Die interventionelle Schmerztherapie sollte, so Dr. Friedli abschliessend, möglichst frühzeitig in die Überlegungen eines Schmerz-Therapiekonzeptes einbezogen werden, denn sie hat in erfahrener und geübter Hand einen ausgesprochen hohen Stellenwert in der Behandlung von komplexen Schmerzzuständen.

\section{Komplementärmedizin}

Eine ganzheitliche Therapie widmet sich neben der Schmerzbehandlung auch der Suche nach den Ursachen der Erkrankung. Traumata, ungünstige Ernährungsgewohnheiten, Stoffwechselprobleme, Umwelteinflüsse, körperliche Fehlbelastungen, Verletzungen, Störungen des Immunsystems sowie Konstitution zählen zu den wichtigsten Faktoren, die in ihrer Summe an der Krankheitsentwicklung beteiligt sein können.

Als Ziel einer Ordnungstherapie gilt es, initial konstitutionelle und konditionelle Schwächen und Belastungen des Patienten $\mathrm{zu}$ identifizieren, um diese unmittelbar und in ihrer Gesamtheit zu behandeln und im weiteren Verlauf meiden zu können. Dr.med. Hans P. Ogal, Abteilung Schmerztherapie an der Aeskulap-Klinik Brunnen, wies in seinem Vortrag darauf hin, dass hierfür meist eine Erweiterung des normalen diagnostischen Spektrums nötig ist. Der Zustand der Ordnung, das heisst, der geistigen, seelischen und körperlichen Ausgeglichenheit des Organismus, lässt sich formal mit Gesundheit gleichsetzen. Krankheit hingegen stellt im weitesten Sinne einen
Zustand der Unordnung im Organismus dar. Neben der Ordnungstherapie kommen komplementärmedizinische Verfahren zur Anwendung, individuell angepasst auf den Patienten und seine Schmerzsymptomatik.

Klassische Homöopathie: Behandelt wird die Gesamtsymptomatik des Krankheitszustandes eines Patienten. Ob hoch potenzierte Homöopathika konstitutionell zum Einsatz kommen oder niedrig potenzierte Mittel vorerst symptomatisch Verwendung finden, muss bei jedem Patienten individuell abgewogen werden. Neben der direkten Schmerzbehandlung können auch psychovegetative Störungen wie Depressivität und Ängstlichkeit mit homöopathischer Medikation behandelt werden. Somit besteht hier zusätzlich der Einfluss auf Faktoren, welche die Schmerzschwelle senken und die Schmerzempfindung steigern können.

Phytotherapie: Sie umfasst externe Applikationen (Kräuterkissen, Phytobalneologie, Segmenttherapie) und die interne Gabe (Tee, standardisierte Pflanzen- und Pflanzenextraktpräparate) zur Prophylaxe, Schmerzbehandlung und Milderung der Nebenwirkungen anderer Verfahren. Arzneipflanzen, die hier zur Anwendung kommen, sind z.B. Arnika, Baldrian, Beinwell, Johanniskraut, Mariendistel, Minzöl, Passionsblume, Pestwurz, Teufelskralle, Weidenrinde und Weihrauch. Auch pflanzliche Enzyme zeigen eine abschwellende und schmerzlindernde Wirkung sowie eine Verbesserung von Rheologie, Durchblutung und Wundheilung.

Orthomolekulare Medizin: Es werden Infusionen mit hoch dosierten Antioxidantien, z.B. die intravenöse Gabe von Vitamin $C$ verwendet. Vitamin $E$ lindert Gelenkschmerzen und ergänzt
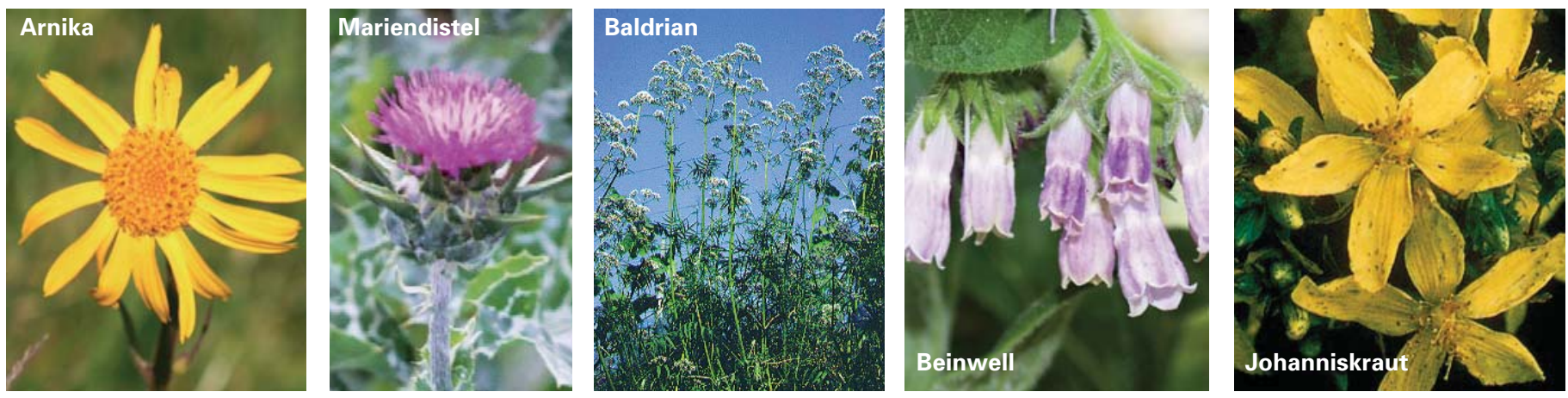
und verbessert die Wirkung der Teufelskralle. Omega-3-Fettsäuren wirken antioxidativ und sind bei rheumatischen Schmerzen indiziert. Bei Nervenschmerzen werden neurotrope Polyvitamine infundiert.

Akupunktur: Hier gilt es, einen gestörten Energiefluss (Qi-Fluss) im Körper wieder herzustellen. Gerade zur Schmerztherapie haben sich auch die Varianten der Mikrosystem-Akupunktur ausserordentlich bewährt, z.B. Ohrakupunktur, Neue Schädelakupunktur nach Yamamoto (YNSA) oder Mundakupunktur nach Gleditsch.

Neuraltherapie: Mit dieser Art der therapeutischen Lokalanästhesie können überempfindliche Körperareale wieder in einen normalen Erregungszustand gebracht werden. Als Umstimmungs-/Gegenirritationsverfahren kann das Lokalanästhetikum im Schmerzbereich auch segmental sub- und intrakutan injiziert werden. Nicht nur die Schmerzperzeption im Bereich des peripheren, sondern auch eine mögliche Plastizität im autonomen Nervensystem sollte bei der Therapie chronischer Schmerzen unbedingt Beachtung finden. Darüber hinaus sollte nach Störfeldern (Narben, chronischen Entzündungen z.B. apikalen Ostitiden, u.a.) gesucht werden, da solche „Störfelder nach Huneke“ im Organismus selber ortsfremde Schmerzen erzeugen oder auch die Schmerzempfindlichkeit stark erhöhen können. Eine weitere Möglichkeit zur Schmerzreduktion bietet die systemische Applikation von Lokalanästhetika.

Blut-/Sauerstofftherapien: Mit ihrer Hilfe kann die Sauerstoffabgabe an das Gewebe über eine Steigerung des 2,3DPG erhöht werden.

Aus- und ableitende Verfahren: Sie sind seit der Antike zur Behandlung bei chronischen Schmerzen bekannt. Bis heute haben die blutentziehenden Techniken (Schröpfen, Aderlass, Blutegeltherapie) und die Derivation (Baunscheidttherapie, Cantharidenpflastertherapie) ihren Einsatz in der Schmerztherapie.

Auch der Einsatz von Wärme resp. die diaphoretische Therapie zählen zu den ausleitenden Verfahren. Besonders Fibromyalgiepatienten und Patienten nach einer Borrelioseinfektion können von einer Hyperthermiebehandlung profitieren. Die Purgation wurde zur Entlastung des Organismus und Reinigung des Darmes durchgeführt. Seit den Erkenntnissen von Prof. Nissle im Jahr 1917 sollte die Sanierung einer Dysbiose zu dem Behandlungsspektrum der Komplementärmedizin gehören. Durch eine gestörte Darmflora und hiermit verbundene nichtoptimale Darmperistaltik wird die Serotoninausschüttung negativ beeinflusst, was sich wiederum negativ auf Schmerzschwelle, Migräneentstehung und die Stimmung der Patienten auswirkt. Es sollte unbedingt eine Normalisierung des intestinalen Milieus und der Bakterienzusammensetzung angestrebt werden.

\section{Psychologische Aspekte: Das Re-Learning-Konzept}

Noch immer ist die therapeutische Versorgung von Schmerzpatienten unzureichend. Das Wissen hingegen über pathophysiologische Vorgänge bei chronischen Schmerzerkrankungen steht dazu ganz im Gegensatz. Chronische Schmerzen zeichnen sich insbesondere dadurch aus, dass sie die typische Mahnfunktion eines Schmerzereignisses vor einem drohenden Schaden verloren haben, so Prof.Dr.med. Christoph Konrad, Institut für Anästhesiologie am Kantonsspital Luzern. Während bei nozizeptiven Schmerzen das schmerzleitende System intakt ist, haben die neuronalen Strukturen bei Neuropathien einen Strukturschaden genommen. Dies führt zu dem elektrophysiologischen Phänomen einer spontanen neuronalen Entleerung sowie zur Freisetzung einer Gruppe von Mediatoren bis hin zur Induktion von Nervenfehlwachstum.

Bisherige therapeutische Ansätze folgten einem multimodalen pharmakologischen und multimodalen extrapharmakologischen Ansatz. Neurountersuchungen haben nun gezeigt, dass durch das Konzept des Re-Learnings ein chronischer Schmerz nachhaltig beeinflussbar ist. Dieses Konzept gilt es nach Einschätzung von Prof. Konrad inskünftig verstärkt zum Einsatz zu bringen. Das könne allerdings nur dann gelingen, wenn Schmerztherapie weiterhin multimodal betrieben und insbesondere der Patient in seiner $\mathrm{Au}$ tonomie gestärkt würde.

\section{Die wichtige Rolle der Zahnmedizin}

Mögliche Zusammenhänge zwischen oralen und allgemeinen Erkrankungen sind in zunehmendem Masse Gegenstand wissenschaftlicher Untersuchungen. Diese dokumentieren, dass nicht nur grössere, sondern auch kleinere orale Infekte andere Erkrankungen des Organismus in Verlauf und Symptomatik negativ zu beeinflussen bzw. deren medizinische Therapie in ihrer Wirksamkeit zu beeinträchtigen vermögen. Wie Prof.Dr.med.dent. Christian E. Besimo, Abteilung für Orale Medizin an der Aeskulap-Klinik Brunnen, den Teilnehmern des Symposiums erläuterte, bestehen beispielsweise zwischen rheumatoider Arthritis und der Zahnbetterkrankung Parodontitis aufgrund der Ähnlichkeit ihrer Pathogenese mögliche Zusammenhänge zwischen Schweregrad der parodontalen und Ausprägung der artikulären Entzündung. Vergleichbare parodontale Einflüsse können auch bei degenerativen Gelenkserkrankungen beobachtet werden.

Die Vielfalt möglicher und belegter Zusammenhänge zwischen Mund- und Allgemeinerkrankungen unterstreicht nach Prof. Besimo insbesondere für den alternden Patienten mit zunehmenden systemischen Defiziten und einer in der Regel längeren zahnmedizinischen Vorgeschichte die dringende Notwendigkeit einer bislang vernachlässigten engen interdisziplinären Vernetzung der medizinisch-zahnärztlichen Diagnostik und Therapie. Diese kann nicht nur bei Schmerzen im Kiefer-, Gesichts- und Kopfbereich, sondern auch bei schmerzhaften Leiden des übrigen Organismus einen wichtigen Schlüssel zur erfolgreichen Diagnostik und Therapie darstellen. Im Rahmen seines Vortrages gab Prof. Besimo einen detaillierten Einblick in die aktuell diskutierten Interaktionen zwischen oralen und systemischen Erkrankungen sowie in die zahnärztliche Schmerzdiagnostik im interdisziplinären Verbund. 